\title{
DESINGING DSP $(0,1)$ ACCEPTANCE SAMPLING PLANS BASED ON TRUNCATED LIFE TESTS UNDER VARIOUS DISTRIBUTIONS USING MINIMUM ANGLE METHOD
}

\author{
A. R. Sudamani Ramaswamy ${ }^{1}$, R. Sutharani ${ }^{2}$ \\ ${ }^{1}$ Associate Professor, Department of Mathematics, Avinashilingam University, Coimbatore - 641043(T.N), India \\ ${ }^{2}$ Assistant professor, Department of Mathematics, Coimbatore Institute of Technology, Coimbatore-641014(T.N), India.
}

\begin{abstract}
In this paper DSP $(0,1)$ sampling plans for truncated life tests are developed using minimum angle method, when the life time of the items follows some selected distributions. The design parameters of the sampling plan are determined for pre-determined acceptance number by satisfying two risks at the specified quality levels simultaneously. The tables of design parameters are provided for various test termination time and mean ratio for some selected distributions. The operating characteristic values are also provided in the table. Some comparisons are made among the selected distributions. The results are explained with examples.
\end{abstract}

Keywords: Probability of acceptance, Rayleigh distribution, generalized exponential distribution, Weibull distribution, Gamma distribution, Producer's risk, Consumer's risk, Minimum angle method.

\section{INTRODUCTION}

Acceptance sampling is a inspecting procedure applied in statistical quality control. Acceptance sampling is a part of operations management and service quality maintenance. It is important for industrial, but also for business purposes helping the decision - making process for the purpose of quality management. In a time- truncated sampling plan, a random sample is selected from a lot of products and put on the test where the number of failures is recorded until the pre specified time. If the number of failures observed is not greater than the specified acceptance number, then the lot will be accepted. Two risks are always attached to an acceptance sampling. The probability of rejecting the good lot is known as the type -1 error (producer's risk) and it is denoted by $\alpha$. The probability of accepting the bad lot is known as the type -2 error (consumer's risk) and it is denoted by $\beta$. An acceptance sampling plan should be designed so that both risks are smaller than the required values. An acceptance sampling plan involves quality contracting on product orders between the producer's risk and consumer's risk. In many practical situations, an important quality characteristic is the lifetime of the product that is, the time over which it fulfills its task. Sampling plans to determine acceptability of a product based on the number of failures recorded until the pre-specified time are called time-truncated sampling plans.
The life tests are discussed by many authors [1] Goode and Kao (1961). Gupta and Groll. [2] Balklizi (2003), [3] Balklizi and EI Masri (2004). [4] Rosaiah and Kantam (2005) and [5] Tsai, Tzong and Shuo (2006). Mohammad Aslam [6] have designed double acceptance sampling plan based on truncated life tests in various distribution. Srinivasa Rao [8] have designed double acceptance sampling plan based on truncated life tests for the Marshall - Olkin extended exponential distribution.

The intent of this paper is to design DSP $(0,1)$ sampling plans for truncated life tests using minimum angle method, when life times of the items follows various distribution.

For designing any sampling inspection plan it is usual practice to select the plan parameters based on the operating characteristic (OC) curve with desired discrimination such that the operating characteristic curve passes through the points with acceptable quality level $\alpha \leq 0.05$ and limiting quality level $\beta \leq 0.10$. Cameron (1952) has used the operating ratio to design the single sampling plan and constructed tables for easy selection of the plan parameters with Poisson model, which is widely, used measure of discrimination to fix the OC curve.

From Cameron table one can observe a jump between the operating ratios of $\mathrm{c}=0$ and $\mathrm{c}=1$ and slow reduction of operating ratios for other values of $\mathrm{c}$.It may also be seen that , in between the OC curves of $\mathrm{c}=0$, and $\mathrm{c}=1$ plans, there is 
vast gap to be filled which leads one to access the possibility of designing plans having OC curves lying between the OC curves of $\mathrm{c}=0$, and $\mathrm{c}=1$ plans . To overcome such situation, Craig (1981) has proposed double sampling plan with acceptance number 0 and 1 and rejection number 2 . Dodge and Romig (1959) have studied the use of DSP - $(0,1)$ plan to product characteristics involving costly and destructive testing.

\subsection{Operating Procedure of Double Sampling Plan of the Type DSP $(0,1)$}

According to Hald (1981), the operating procedure for DSP (0, 1) is as follows:

(i) From a lot, select a sample size $\mathrm{n}_{1}$, and observe the number of defectives $d_{1}$.

(ii) If $\mathrm{d}_{1}=0$, accept the lot. If $\mathrm{d}_{1}>1$, reject the lot.

(iii) If $\mathrm{d}_{1}=1$, select a second sample of size $\mathrm{n}_{2}$ and observe $\mathrm{d}_{2}$.

If $\mathrm{d}_{2}=0$, accept the lot. Otherwise reject the lot.

\subsubsection{Operating Procedure of Double Sampling Plan} of the Type DSP $(0,1)$ for Life Tests

(i) From a lot, select a sample size $\mathrm{n}_{1}$, and observe the number of defectives $d_{1}$, during the time $t_{0}$.

(ii) If $\mathrm{d}_{1}=0$, accept the lot. If $\mathrm{d}_{1}>1$, reject the lot.

(iii) If $\mathrm{d}_{1}=1$, select a second sample of size $\mathrm{n}_{2}$ and observe $\mathrm{d}_{2}$ during the time $\mathrm{t}_{0}$.. If $\mathrm{d}_{2}=0$, accept the lot. Otherwise reject the lot.

\subsection{PROPERTIES}

1. Fixing $\mathrm{n}_{1}=\mathrm{n}$ and $\mathrm{n}_{2}=\mathrm{kn}(\mathrm{k}>0)$, the $\mathrm{OC}$ function of DSP - $(0,1)$ plan is identical to the OC function of ChSP - 1 plan.

2. When $\mathrm{k}=0$ and $\mathrm{k}=\infty$, the OC function of DSP $-(0$, 1) plan reduces to the $\mathrm{OC}$ functions of single sampling plans with $\mathrm{c}=0$ and $\mathrm{c}=1$ respectively.

3. The OC curves of DSP - $(0,1)$ plans are lying between those of $\mathrm{c}=0$ and $\mathrm{c}=1$ single sampling plans.

The following is the operating procedure for $\operatorname{DSP}(0,1)$ for life test in the form of a flow chart.

\section{FLOWCHART}

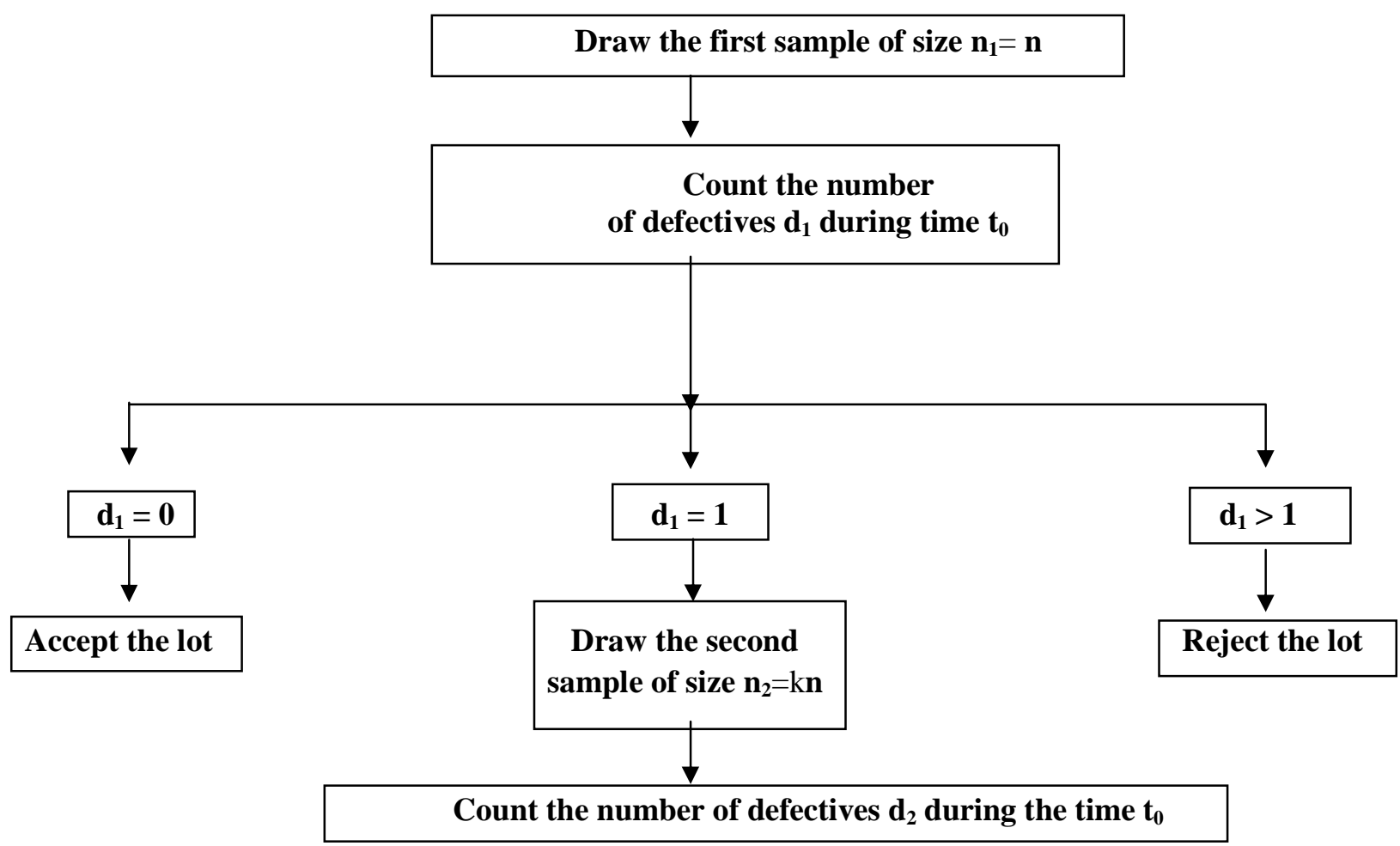




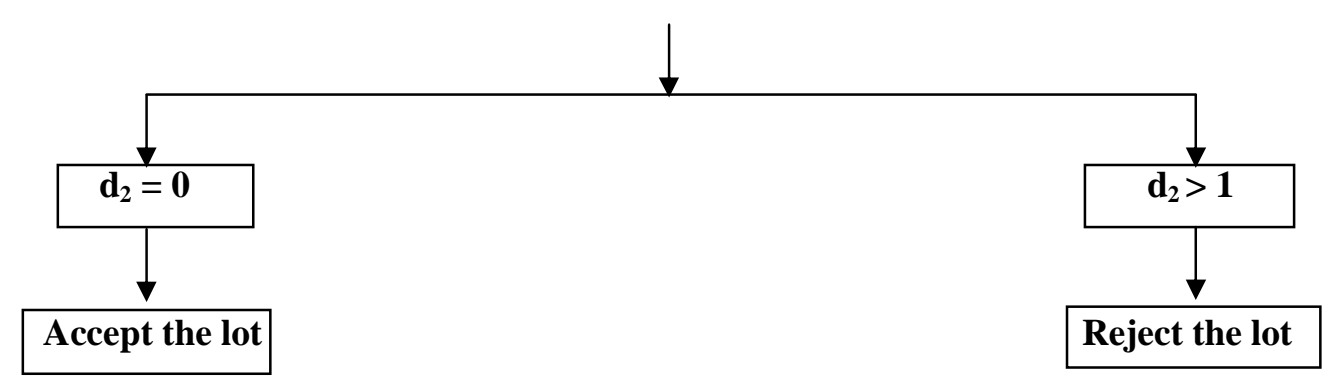

The DSP $(0,1)$ is composed of parameters $n_{1}$ and $n_{2}$ if $t_{0}$ is specified. Let $\lambda$ be the unknown average life and $\lambda_{0}$ be the specified average life. A lot is considered to be good if the true unknown average life is more than the specified average life. In this paper we have applied Rayleigh, Generalised exponential, Weibull and Gamma distribution to design DSP $(0,1)$ plan for life test.We assume that the lot size is large enough to use the binomial distribution to find the probability of acceptance of the lot. Then the probability of acceptance for $\operatorname{DSP}(0,1)$ is given by

$\mathrm{P}(\mathrm{A})=\mathrm{P}($ no failure occur in sample 1$)+\mathrm{P}(1$ failure occur in sample 1 and 0 failure occur in sample 2). Under the conditions for application of binomial model for the OC function of DSP - $(0,1)$ or the probability of acceptance DSP $(0,1)$ is given by,

$$
L(p)=(1-p)^{n_{1}}+n_{1} p(1-p)^{\left(n_{1}+n_{2}-1\right)}
$$

The usual practice is to choose the second sample size equals to some constant $(\mathrm{k})$ multiple of first sample size which facilitates sample administration of the $\operatorname{DSP}(0,1)$ sampling plan.

Taking $\mathrm{n}_{1}=\mathrm{n}$ and $\mathrm{n}_{2}=\mathrm{kn}$ we get

$$
L(p)=(1-p)^{n}+\mathrm{np}(1-p)^{(n(k+1)-1)}
$$

The failure probability of an item by time $t_{0}$ is given by

$$
\mathbf{P}=\mathbf{F}\left(\mathbf{t}_{\mathbf{0}}, \lambda\right)
$$

\section{RAYLEIGH DISTRIBUTION:}

The Cumulative Distribution Function (CDF) is given as,

$$
F(t, \lambda)=1-e^{-\frac{1}{2}\left(\frac{t}{\lambda}\right)^{2}}
$$

Where, $\lambda$ is the scale parameter

\section{GENERALIZED}

EXPONENTIAL

\section{DISTRIBUTION}

The cumulative distribution function (cdf) of the exponential distribution is given by

$$
F(t, \lambda)=\left(1-e^{-\frac{t}{\lambda}}\right)^{\alpha}
$$

Where $\lambda$ is the scale parameter and $\alpha$ is the shape parameter and it is equal to 2

\section{WEIBULL DISTRIBUTION:}

The cumulative distribution function (cdf) of the weibull distribution is given by

$$
F(t, \lambda)=1-e^{-\left(\frac{t}{\lambda}\right)^{m}}
$$

Where $\lambda$ is the scale parameter and $m$ is the shape parameter and it is equal to 2

\section{GAMMA DISTRIBUTION:}

The cumulative distribution function (cdf) of the exponential distribution is given by

$$
F(t, \lambda)=1-e^{-\frac{t}{\lambda}} \sum_{j=0}^{\gamma-1}\left(\frac{t}{\lambda}\right)^{j} / j !
$$

Where $\lambda$ is the scale parameter and $\gamma$ is the shape parameter and it is equal to 2

The probability of acceptance can be regarded as a function of the deviation of the unknown average life $\lambda_{0}$ from its specified average life $\lambda$. By fixing the time 
termination ratio $t / \lambda_{0}$ as $0.628,0.942,1.257,2.356,3.141$, 3.927, 4.712 and the mean ratios $\lambda \lambda_{0}=4,6,8,10$ and 12 , producers risk $\alpha \leq 0.05$ and consumers risk $\beta \leq 0.10$ one can find the parameters.

\section{NOTATION}

$\begin{array}{ll}\mathrm{n} & - \text { Sample size } \\ \mathrm{c} & - \text { Acceptance number } \\ \mathrm{t}_{0} & - \text { Termination time } \\ \alpha & - \text { Producer's risk } \\ \beta & - \text { Consumer's risk } \\ \mathrm{P} & - \text { Failure probability } \\ \mathrm{L}(\mathrm{p}) & - \text { Probability of acceptance } \\ \lambda & - \text { Mean life } \\ \lambda_{0} & - \text { Specified life } \\ \theta & - \text { Minimum angle } \\ \mathrm{d} & - \text { Number of defectives }\end{array}$

\section{MINIMUM ANGLE METHOD}

The practical performance of a sampling plan is revealed by it operating characteristic curve. Norman Bush et. al. [7] have used different techniques involving comparison of some portion of the OC curve to that of the ideal curve. The approach of minimum angle method by considering the tangent of the angle between the lines joining the points $(\mathrm{AQL}, 1-\alpha) \&(\mathrm{LQL}, \beta)$ is shown in Figure where $\mathrm{p}_{1}=\mathrm{AQL}$, $\mathrm{p}_{2}=$ LQL. By employing this method one can get a better discriminating plan with the minimum angle. Tangent of angle made by lines $\mathrm{AB}$ and $\mathrm{AC}$ is

\section{$\operatorname{Tan} \theta=B C / A C$}

$\operatorname{Tan} \theta=\left(\mathbf{p}_{2}-\mathbf{p}_{1}\right) /\left(\mathbf{P a}\left(\mathbf{p}_{1}\right)-\mathbf{P a}\left(\mathbf{p}_{2}\right)\right)$

(6)

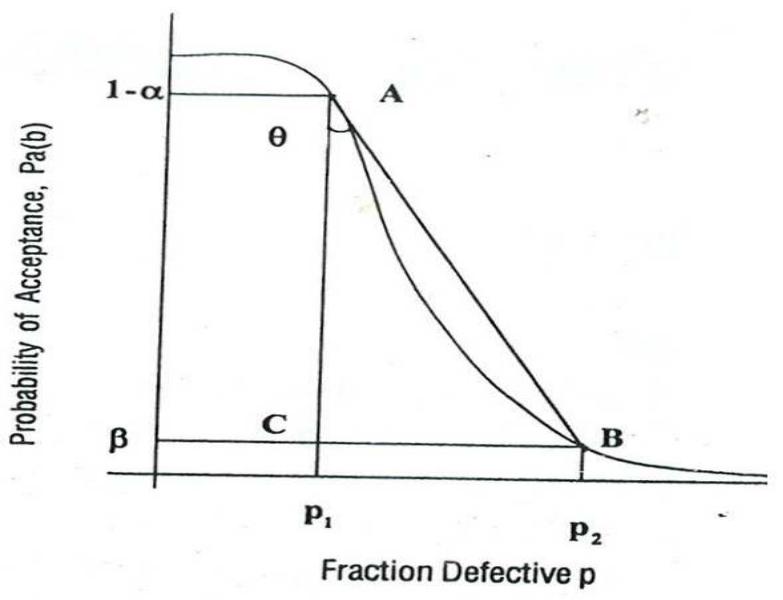

Minimum angle for given $p_{1}$ and $p_{2}$
The smaller the value of this $\tan \theta$, closer is the angle $\theta$ approaching zero and the chord $\mathrm{AB}$ approaching $\mathrm{AC}$, the ideal condition through (AQL, 1- $\alpha$ ). This criterion minimizes simultaneously the consumer's and producer's risks. Thus both the producer and consumer favour the plans evolved by the criterion.

In this paper we design parameters of the DSP $(0,1)$ plan based on truncated life tests for various distributions, using minimum angle method. The minimum angle method of the DSP $(0,1)$ sampling plan under various distributions for truncated life test is given below.

\section{CONSTRUCTION OF TABLES}

The Tables are constructed using OC function for DSP $(0,1)$ sampling plans under various distributions. The test termination ratio $t / \lambda_{0}$ values are fixed as $0.628,0.912,1.257$, $1.571,2.356,3.141,3.927$ and 4.712 , and the mean ratio $\lambda / \lambda_{0}$ values are fixed as $4,6,8,10,12$. For various time ratios $t / \lambda_{0}$ and mean ratios $\lambda / \lambda_{0}$ the parameter values $\mathrm{n}_{1}$ and $\mathrm{n}_{2}$ are obtained and satisfying $\mathrm{L}\left(\mathrm{p}_{1}\right) \geq 0.95$ and $\mathrm{L}\left(\mathrm{p}_{2}\right) \leq 0.10$ for various distribution and are provided in Table 1 to Table 4 . The value $\theta$ and $\tan \theta$ values are also provided in each table. The parameters can be selected such that the angle is minimum.

\section{EXAMPLES}

Assume that an experimenter wants to establish that the lifetime of the electrical devices produced in the factory ensures that the true unknown mean life is at least 1000 hours when the ratio of the unknown average life is 4 . Following are the results obtained when the lifetime of the test items follows the Rayleigh, generalized exponential distribution, Weibull distribution, Gamma distribution respectively.

\subsection{Rayleigh Distribution}

Let the distribution followed be Rayleigh. Here we get the sample size as $n=15$ and $\mathrm{k}=2$. The lot is accepted at given mean ratio $\lambda / \lambda_{0}=6$ during 628 hours which satisfies the condition of producer's risk and consumer's risk $\alpha \leq 0.05, \beta$ $\leq 0.10$, from the Table 1 , one can observe that the minimum angle is $\theta=10.54134^{\circ}$ and also $\alpha=0.0097$ and $\beta=0.057$ which is very much less than the specified risk. Thus the required DSP $(0,1)$ plan has parameters $(15,30)$ which satisfies both the producers risk and consumer's risk.

\subsection{Generalized Exponential Distribution}

Let the distribution followed be generalized exponential. Here we get the sample size as $\mathrm{n}=10$ and $\mathrm{k}=3$. The lot is accepted at given mean ratio $\lambda / \lambda_{0}=6$ during 628 hours which satisfies the condition of producer's risk and consumer's risk $\alpha$ $\leq 0.05, \beta \leq 0.10$, from the Table 2 , one can observe that the minimum angle is $\theta=13.12339^{\circ}$ and also $\alpha=.0225$ and $\beta=$ 
.086 which is very much less than the specified risk. Thus the required DSP $(0,1)$ plan has parameters $(10,30)$ which satisfies both the producers risk and consumer's risk.

\subsection{Weibull Distribution}

Let the distribution followed be Weibull. Here we get the sample size as $n=7$ and $\mathrm{k}=2$. The lot is accepted at given mean ratio $\lambda / \lambda_{0}=6$ during 628 hours which satisfies the condition of producer's risk and consumer's risk $\alpha \leq 0.05, \beta \leq$ 0.10 , from the Table 3 , one can observe that the minimum angle is $\quad \theta=18.87288^{\circ}$ and also $\alpha=.009$ and $\beta=.069$ which is very much less than the specified risk. Thus the required DSP $(0,1)$ plan has parameters $(7,14)$ which satisfies both the producers risk and consumer's risk.

\subsection{Gamma Distribution}

Let the distribution followed be Gamma. Here we get the sample size as $n=18$ and $\mathrm{k}=2$. The lot is accepted at given mean ratio $\lambda / \lambda_{0}=6$ during 628 hours which satisfies the condition of producer's risk and consumer's risk $\alpha \leq 0.05, \beta \leq$ 0.10 , from the Table 4 , one can observe that the minimum angle is $\quad \theta=8.015064^{\circ}$ and also $\alpha=.0119$ and $\beta=.092$ which is very much less than the specified risk. Thus the required DSP $(0,1)$ plan has parameters $(18,36)$ which satisfies both the producers risk and consumer's risk.

Table - 1: Sample size for DSP $(0,1)$ using Minimum Angle Method for specified values of k when the life time of the items follows Rayleigh Distribution

\begin{tabular}{|c|c|c|c|c|c|c|c|}
\hline $\mathbf{t} / \boldsymbol{\lambda}_{\mathbf{0}}$ & $\lambda / \boldsymbol{\lambda}_{\mathbf{0}}$ & $\mathbf{k}$ & $\mathbf{n}$ & $\mathbf{L}\left(\mathbf{p}_{\mathbf{1}}\right)$ & $\mathbf{L}\left(\mathbf{p}_{\mathbf{2}}\right)$ & $\mathbf{T a n} \boldsymbol{\theta}$ & $\boldsymbol{\Theta}$ \\
\hline 0.628 & 4 & 2 & 15 & 0.956604 & 0.057872 & 0.185504 & 10.50913 \\
\hline 0.628 & 4 & 1 & 20 & 0.972999 & 0.088719 & 0.188536 & 10.67696 \\
\hline 0.628 & 4 & 3 & 12 & 0.954543 & 0.095023 & 0.193967 & 10.97715 \\
\hline 0.942 & 4 & 1 & 10 & 0.965085 & 0.054237 & 0.363377 & 19.96997 \\
\hline 0.942 & 4 & 2 & 6 & 0.961153 & 0.076524 & 0.374147 & 20.51319 \\
\hline 1.257 & 4 & 1 & 5 & 0.969421 & 0.071827 & 0.554805 & 29.02171 \\
\hline 1.257 & 4 & 2 & 3 & 0.964615 & 0.099971 & 0.575947 & 29.93966 \\
\hline 1.571 & 4 & 1 & 4 & 0.952636 & 0.027549 & 0.686047 & 34.45195 \\
\hline 1.571 & 4 & 2 & 3 & 0.923221 & 0.025049 & 0.706606 & 35.24525 \\
\hline 0.628 & 6 & 1 & 20 & 0.994152 & 0.088719 & 0.191626 & 10.84785 \\
\hline $\mathbf{0 . 6 2 8}$ & $\mathbf{6}$ & $\mathbf{2}$ & $\mathbf{1 5}$ & $\mathbf{0 . 9 9 0 2 6 4}$ & $\mathbf{0 . 0 5 7 8 7 2}$ & $\mathbf{0 . 1 8 6 0 8 5}$ & $\mathbf{1 0 . 5 4 1 3 4}$ \\
\hline 0.628 & 6 & 3 & 12 & 0.989616 & 0.095023 & 0.193948 & 10.97612 \\
\hline 0.942 & 6 & 1 & 9 & 0.993676 & 0.077916 & 0.377918 & 20.70246 \\
\hline 0.942 & 6 & 2 & 6 & 0.991334 & 0.076524 & 0.37831 & 20.72212 \\
\hline 1.257 & 6 & 1 & 5 & 0.993332 & 0.071827 & 0.569135 & 29.6457 \\
\hline 1.257 & 6 & 2 & 3 & 0.99213 & 0.099971 & 0.587855 & 30.44935 \\
\hline 1.257 & 6 & 3 & 3 & 0.987437 & 0.093749 & 0.586849 & 30.40652 \\
\hline 1.571 & 6 & 1 & 3 & 0.993489 & 0.077142 & 0.73682 & 36.38354 \\
\hline 1.571 & 6 & 2 & 3 & 0.981802 & 0.025049 & 0.705703 & 35.21072 \\
\hline 2.356 & 6 & 1 & 2 & 0.984301 & 0.003886 & 0.880726 & 41.3712 \\
\hline 2.356 & 6 & 2 & 2 & 0.958039 & 0.01117 & 0.911928 & 42.36257 \\
\hline 3.141 & 6 & 1 & 2 & 0.955007 & 0.000155 & 0.905629 & 42.16491 \\
\hline 3.141 & 6 & 2 & 1 & 0.956838 & 0.007206 & 0.910607 & 42.32122 \\
\hline 3.927 & 6 & 1 & 1 & 0.962828 & 0.000896 & 0.838678 & 39.98581 \\
\hline 0.628 & 8 & 1 & 20 & 0.998088 & 0.088719 & 0.19342 & 10.94699 \\
\hline 0.628 & 8 & 2 & 13 & 0.997538 & 0.088371 & 0.193464 & 10.94938 \\
\hline
\end{tabular}




\begin{tabular}{|c|c|c|c|c|c|c|c|}
\hline 0.628 & 8 & 3 & 12 & 0.996539 & 0.095023 & 0.195105 & 11.04002 \\
\hline 0.942 & 8 & 1 & 9 & 0.99793 & 0.077916 & 0.381975 & 20.90559 \\
\hline 0.942 & 8 & 2 & 6 & 0.997137 & 0.076524 & 0.381726 & 20.89316 \\
\hline 0.942 & 8 & 3 & 6 & 0.995343 & 0.070103 & 0.379817 & 20.79764 \\
\hline 1.257 & 8 & 1 & 5 & 0.997815 & 0.071827 & 0.576571 & 29.96651 \\
\hline 1.257 & 8 & 2 & 3 & 0.997403 & 0.099971 & 0.594918 & 30.74916 \\
\hline 1.257 & 8 & 3 & 3 & 0.995777 & 0.093749 & 0.591887 & 30.62074 \\
\hline 1.571 & 8 & 1 & 3 & 0.997867 & 0.077142 & 0.749174 & 36.83961 \\
\hline 1.571 & 8 & 2 & 4 & 0.989991 & 0.007225 & 0.70188 & 35.06424 \\
\hline 2.356 & 8 & 1 & 2 & 0.99475 & 0.000242 & 0.900179 & 41.99288 \\
\hline 2.356 & 8 & 2 & 2 & 0.985256 & 0.000019 & 0.964629 & 43.96856 \\
\hline 3.141 & 8 & 1 & 1 & 0.994497 & 0.014359 & 0.937228 & 43.14409 \\
\hline 3.141 & 8 & 2 & 1 & 0.984686 & 0.007206 & 0.939777 & 43.22174 \\
\hline 3.927 & 8 & 1 & 1 & 0.987117 & 0.000896 & 0.898427 & 41.93738 \\
\hline 3.927 & 8 & 2 & 1 & 0.965571 & 0.000448 & 0.918067 & 42.55401 \\
\hline 4.712 & 8 & 1 & 1 & 0.97464 & 0.000002 & 0.862638 & 40.78231 \\
\hline 0.628 & 10 & 1 & 20 & 0.999205 & 0.088719 & 0.194398 & 11.00099 \\
\hline 0.628 & 10 & 2 & 13 & 0.998973 & 0.088371 & 0.194374 & 10.99963 \\
\hline 0.628 & 10 & 3 & 12 & 0.998548 & 0.095023 & 0.195896 & 11.08366 \\
\hline 0.942 & 10 & 1 & 9 & 0.999139 & 0.077916 & 0.384167 & 21.01514 \\
\hline 0.942 & 10 & 2 & 6 & 0.998804 & 0.076524 & 0.383727 & 20.99315 \\
\hline 0.942 & 10 & 3 & 6 & 0.998037 & 0.070103 & 0.381389 & 20.87629 \\
\hline 1.257 & 10 & 1 & 5 & 0.99909 & 0.071827 & 0.580523 & 30.13613 \\
\hline 1.257 & 10 & 2 & 3 & 0.998915 & 0.099971 & 0.59881 & 30.91362 \\
\hline 1.257 & 10 & 3 & 3 & 0.99822 & 0.093749 & 0.595152 & 30.75906 \\
\hline 1.571 & 10 & 1 & 3 & 0.999112 & 0.077142 & 0.755573 & 37.07372 \\
\hline 1.571 & 10 & 2 & 4 & 0.995738 & 0.007225 & 0.704711 & 35.17277 \\
\hline 1.571 & 10 & 3 & 2 & 0.997845 & 0.077142 & 0.756614 & 37.11165 \\
\hline 2.356 & 10 & 1 & 2 & 0.997793 & 0.01117 & 0.922643 & 42.69596 \\
\hline 2.356 & 10 & 2 & 2 & 0.993656 & 0.003886 & 0.91971 & 42.60507 \\
\hline 3.141 & 10 & 1 & 1 & 0.997683 & 0.014359 & 0.960682 & 43.85119 \\
\hline 3.141 & 10 & 2 & 1 & 0.993379 & 0.007206 & 0.957907 & 43.76839 \\
\hline 3.141 & 10 & 3 & 2 & 0.970927 & 0.000019 & 0.973001 & 44.21599 \\
\hline 3.927 & 10 & 1 & 1 & 0.994493 & 0.000896 & 0.931306 & 42.96293 \\
\hline 3.927 & 10 & 2 & 1 & 0.984675 & 0.000448 & 0.940173 & 43.23378 \\
\hline 3.927 & 10 & 3 & 1 & 0.97626 & 0.000448 & 0.94828 & 43.47937 \\
\hline 4.712 & 10 & 1 & 1 & 0.988959 & 0.000002 & 0.904929 & 42.14285 \\
\hline 4.712 & 10 & 2 & 1 & 0.970236 & 0.000051 & 0.922378 & 42.68775 \\
\hline 0.628 & 12 & 1 & 20 & 0.999613 & 0.088719 & 0.194972 & 11.03264 \\
\hline 0.628 & 12 & 2 & 13 & 0.9995 & 0.088371 & 0.194921 & 11.02987 \\
\hline
\end{tabular}




\begin{tabular}{|l|l|l|l|c|c|c|c|}
\hline 0.628 & 12 & 3 & 12 & 0.99929 & 0.095023 & 0.1964 & 11.11149 \\
\hline 0.942 & 12 & 1 & 9 & 0.999581 & 0.077916 & 0.385448 & 21.07907 \\
\hline 0.942 & 12 & 2 & 6 & 0.999417 & 0.076524 & 0.384935 & 21.05348 \\
\hline 0.942 & 12 & 3 & 6 & 0.999038 & 0.070103 & 0.382432 & 20.92843 \\
\hline 1.257 & 12 & 1 & 5 & 0.999557 & 0.071827 & 0.582815 & 30.23427 \\
\hline 1.257 & 12 & 2 & 3 & 0.999471 & 0.099971 & 0.601106 & 31.01033 \\
\hline 1.257 & 12 & 3 & 3 & 0.999128 & 0.093749 & 0.597203 & 30.84578 \\
\hline 1.571 & 12 & 1 & 3 & 0.999568 & 0.025049 & 0.71866 & 35.70328 \\
\hline 1.571 & 12 & 2 & 3 & 0.998727 & 0.025049 & 0.71928 & 35.72672 \\
\hline 2.356 & 12 & 1 & 2 & 0.998921 & 0.01117 & 0.929976 & 42.92208 \\
\hline 2.356 & 12 & 2 & 3 & 0.993859 & 0.000242 & 0.924486 & 42.75295 \\
\hline 3.141 & 12 & 1 & 1 & 0.998866 & 0.014359 & 0.974212 & 44.25161 \\
\hline 3.141 & 12 & 2 & 1 & 0.996711 & 0.007206 & 0.969291 & 44.1066 \\
\hline 3.927 & 12 & 1 & 1 & 0.997282 & 0.000896 & 0.950851 & 43.55681 \\
\hline 3.927 & 12 & 2 & 2 & 0.978225 & 0.000001 & 0.968503 & 44.08332 \\
\hline 4.712 & 12 & 1 & 1 & 0.994495 & 0.000002 & 0.930941 & 42.95173 \\
\hline 4.712 & 12 & 2 & 1 & 0.98468 & 0.000051 & 0.940207 & 43.23482 \\
\hline 4.712 & 12 & 3 & 1 & 0.976267 & 0.000051 & 0.948309 & 43.48022 \\
\hline
\end{tabular}

Table - 2: Sample size for DSP (0,1) using Minimum Angle Method for specified values of k when the life time of the items follows Generalized Exponential Distribution

\begin{tabular}{|c|c|c|c|c|c|c|c|}
\hline $\mathbf{t} / \boldsymbol{\lambda}_{\mathbf{0}}$ & $\boldsymbol{\lambda} / \boldsymbol{\lambda}_{\mathbf{0}}$ & $\mathbf{k}$ & $\mathbf{n}$ & $\mathbf{L}\left(\mathbf{p}_{\mathbf{1}}\right)$ & $\mathbf{L}\left(\mathbf{p}_{\mathbf{2}}\right)$ & $\tan \boldsymbol{\theta}$ & $\boldsymbol{\Theta}$ \\
\hline 0.628 & 4 & 1 & 16 & 0.950865 & 0.088555 & 0.227718 & 12.82855 \\
\hline 0.628 & 6 & 1 & 16 & 0.987981 & 0.088555 & 0.230813 & 12.99701 \\
\hline 0.628 & 6 & 2 & 10 & 0.985696 & 0.098711 & 0.23405 & 13.17298 \\
\hline $\mathbf{0 . 6 2 8}$ & $\mathbf{6}$ & $\mathbf{3}$ & $\mathbf{1 0}$ & $\mathbf{0 . 9 7 7 4 0 4}$ & $\mathbf{0 . 0 8 6 9 4 6}$ & $\mathbf{0 . 2 3 3 1 3 8}$ & $\mathbf{1 3 . 1 2 3 3 9}$ \\
\hline 0.942 & 6 & 1 & 8 & 0.985463 & 0.095894 & 0.39477 & 21.54261 \\
\hline 0.942 & 6 & 2 & 6 & 0.975817 & 0.066422 & 0.386163 & 21.11474 \\
\hline 1.257 & 6 & 1 & 5 & 0.982608 & 0.098585 & 0.538681 & 28.31052 \\
\hline 1.257 & 6 & 2 & 4 & 0.967578 & 0.059963 & 0.524679 & 27.68504 \\
\hline 1.257 & 6 & 3 & 4 & 0.950448 & 0.056834 & 0.532899 & 28.05312 \\
\hline 1.571 & 6 & 1 & 4 & 0.974711 & 0.067565 & 0.633256 & 32.34427 \\
\hline 1.571 & 6 & 2 & 3 & 0.957787 & 0.05355 & 0.635294 & 32.42753 \\
\hline 2.356 & 6 & 1 & 2 & 0.96898 & 0.086077 & 0.808616 & 38.95956 \\
\hline 3.141 & 6 & 1 & 2 & 0.926396 & 0.020265 & 0.82691 & 39.58767 \\
\hline 3.141 & 6 & 2 & 1 & 0.930217 & 0.085164 & 0.886676 & 41.56263 \\
\hline 0.628 & 8 & 1 & 16 & 0.995826 & 0.088555 & 0.23342 & 13.13871 \\
\hline 0.628 & 8 & 2 & 10 & 0.994992 & 0.098711 & 0.236282 & 13.29412 \\
\hline 0.628 & 8 & 3 & 10 & 0.991912 & 0.086946 & 0.234014 & 13.17102 \\
\hline 0.942 & 8 & 1 & 8 & 0.994826 & 0.095894 & 0.400416 & 21.82197 \\
\hline
\end{tabular}




\begin{tabular}{|c|c|c|c|c|c|c|c|}
\hline 0.942 & 8 & 2 & 6 & 0.991211 & 0.066422 & 0.38922 & 21.26701 \\
\hline 0.942 & 8 & 3 & 5 & 0.989764 & 0.098135 & 0.403696 & 21.98373 \\
\hline 1.257 & 8 & 1 & 5 & 0.993664 & 0.098585 & 0.54832 & 28.73682 \\
\hline 1.257 & 8 & 2 & 4 & 0.987849 & 0.059963 & 0.528933 & 27.87583 \\
\hline 1.257 & 8 & 3 & 4 & 0.980778 & 0.056834 & 0.531189 & 27.97675 \\
\hline 1.571 & 8 & 1 & 4 & 0.990522 & 0.067565 & 0.645461 & 32.84066 \\
\hline 1.571 & 8 & 2 & 3 & 0.983698 & 0.05355 & 0.640471 & 32.63838 \\
\hline 2.356 & 8 & 1 & 2 & 0.987847 & 0.086077 & 0.836483 & 39.91192 \\
\hline 2.356 & 8 & 2 & 2 & 0.967053 & 0.032935 & 0.807517 & 38.92146 \\
\hline 3.141 & 8 & 1 & 1 & 0.988882 & 0.16206 & 0.979593 & 44.40937 \\
\hline 3.141 & 8 & 2 & 1 & 0.970039 & 0.085164 & 0.915325 & 42.46865 \\
\hline 3.927 & 8 & 1 & 1 & 0.977358 & 0.076512 & 0.89972 & 41.97836 \\
\hline 4.712 & 8 & 1 & 1 & 0.960744 & 0.035465 & 0.847287 & 40.27418 \\
\hline 0.628 & 10 & 1 & 16 & 0.998201 & 0.088555 & 0.235003 & 13.22473 \\
\hline 0.628 & 10 & 2 & 10 & 0.997833 & 0.098711 & 0.237754 & 13.37398 \\
\hline 0.628 & 10 & 3 & 10 & 0.996462 & 0.086946 & 0.235037 & 13.22655 \\
\hline 0.942 & 10 & 1 & 8 & 0.997736 & 0.095894 & 0.403845 & 21.99107 \\
\hline 0.942 & 10 & 2 & 6 & 0.996114 & 0.066422 & 0.391747 & 21.39261 \\
\hline 0.942 & 10 & 3 & 5 & 0.995441 & 0.098135 & 0.405886 & 22.09157 \\
\hline 1.257 & 10 & 1 & 5 & 0.997187 & 0.098585 & 0.554171 & 28.99395 \\
\hline 1.257 & 10 & 2 & 4 & 0.994528 & 0.059963 & 0.532846 & 28.05072 \\
\hline 1.257 & 10 & 3 & 4 & 0.991191 & 0.056834 & 0.532964 & 28.056 \\
\hline 1.571 & 10 & 1 & 4 & 0.995719 & 0.067565 & 0.653325 & 33.15759 \\
\hline 1.571 & 10 & 2 & 3 & 0.992524 & 0.05355 & 0.645797 & 32.85424 \\
\hline 1.571 & 10 & 3 & 3 & 0.988052 & 0.051714 & 0.647614 & 32.92767 \\
\hline 2.356 & 10 & 1 & 2 & 0.994347 & 0.086077 & 0.853634 & 40.4852 \\
\hline 2.356 & 10 & 2 & 2 & 0.984166 & 0.032935 & 0.815081 & 39.18282 \\
\hline 3.141 & 10 & 1 & 1 & 0.994721 & 0.16206 & 1.012095 & 45.3444 \\
\hline 3.141 & 10 & 2 & 1 & 0.985285 & 0.085164 & 0.936242 & 43.11401 \\
\hline 3.141 & 10 & 3 & 1 & 0.97717 & 0.084613 & 0.944176 & 43.35531 \\
\hline 3.927 & 10 & 1 & 1 & 0.988875 & 0.076512 & 0.937684 & 43.158 \\
\hline 3.927 & 10 & 2 & 1 & 0.970022 & 0.039074 & 0.918965 & 42.58192 \\
\hline 3.927 & 10 & 3 & 1 & 0.954936 & 0.039017 & 0.934044 & 43.04682 \\
\hline 4.712 & 10 & 1 & 1 & 0.980067 & 0.035465 & 0.89024 & 41.67674 \\
\hline 0.628 & 12 & 1 & 16 & 0.999104 & 0.088555 & 0.235984 & 13.27796 \\
\hline 0.628 & 12 & 2 & 10 & 0.998919 & 0.098711 & 0.238695 & 13.425 \\
\hline 0.628 & 12 & 3 & 10 & 0.998224 & 0.086946 & 0.235795 & 13.26772 \\
\hline 0.942 & 12 & 1 & 8 & 0.998861 & 0.095894 & 0.40598 & 22.09614 \\
\hline 0.942 & 12 & 2 & 6 & 0.998034 & 0.066422 & 0.393496 & 21.47946 \\
\hline 0.942 & 12 & 3 & 5 & 0.997684 & 0.098135 & 0.407522 & 22.17199 \\
\hline
\end{tabular}




\begin{tabular}{|c|c|c|c|c|c|c|c|}
\hline 1.257 & 12 & 1 & 5 & 0.998571 & 0.098585 & 0.557832 & 29.15419 \\
\hline 1.257 & 12 & 2 & 4 & 0.997198 & 0.059963 & 0.535662 & 28.17625 \\
\hline 1.257 & 12 & 3 & 4 & 0.995444 & 0.056834 & 0.534877 & 28.1413 \\
\hline 1.571 & 12 & 1 & 4 & 0.9978 & 0.067565 & 0.658397 & 33.36079 \\
\hline 1.571 & 12 & 2 & 3 & 0.996125 & 0.05355 & 0.649778 & 33.01492 \\
\hline 2.356 & 12 & 1 & 2 & 0.997035 & 0.086077 & 0.864598 & 40.84664 \\
\hline 2.356 & 12 & 2 & 2 & 0.991534 & 0.032935 & 0.821629 & 39.40751 \\
\hline 3.141 & 12 & 1 & 1 & 0.997187 & 0.16206 & 1.032602 & 45.91893 \\
\hline 3.141 & 12 & 2 & 1 & 0.992001 & 0.085164 & 0.950947 & 43.55971 \\
\hline 3.927 & 12 & 1 & 1 & 0.993932 & 0.076512 & 0.962577 & 43.90761 \\
\hline 3.927 & 12 & 2 & 2 & 0.954136 & 0.001523 & 0.927016 & 42.83101 \\
\hline 4.712 & 12 & 1 & 1 & 0.988878 & 0.035465 & 0.919483 & 42.59802 \\
\hline 4.712 & 12 & 2 & 1 & 0.97003 & 0.017898 & 0.920721 & 42.63642 \\
\hline 4.712 & 12 & 3 & 1 & 0.954947 & 0.017893 & 0.935535 & 43.09242 \\
\hline
\end{tabular}

Table - 3: Sample size for DSP $(0,1)$ using Minimum Angle Method for specified values of k when the life time of the items follows Weibull Distribution

\begin{tabular}{|c|c|c|c|c|c|c|c|}
\hline $\mathbf{t} / \boldsymbol{\lambda}_{\boldsymbol{0}}$ & $\boldsymbol{\lambda} \boldsymbol{\lambda}_{\boldsymbol{0}}$ & $\mathbf{k}$ & $\mathbf{n}$ & $\mathbf{L}\left(\mathbf{p}_{\mathbf{1}}\right)$ & $\mathbf{L}\left(\mathbf{p}_{\mathbf{2}}\right)$ & $\tan \boldsymbol{\theta}$ & $\boldsymbol{\Theta}$ \\
\hline 0.628 & 4 & 2 & 7 & 0.959278 & 0.0694 & 0.338875 & 18.72023 \\
\hline 0.628 & 4 & 1 & 10 & 0.971826 & 0.082513 & 0.33909 & 18.73129 \\
\hline 0.628 & 4 & 3 & 6 & 0.951752 & 0.094561 & 0.351797 & 19.3817 \\
\hline 0.942 & 4 & 1 & 4 & 0.973909 & 0.096367 & 0.608871 & 31.33604 \\
\hline 0.942 & 4 & 2 & 3 & 0.956501 & 0.073342 & 0.604999 & 31.17389 \\
\hline 1.257 & 4 & 1 & 3 & 0.953363 & 0.029551 & 0.757731 & 37.15233 \\
\hline 0.628 & 6 & 1 & 10 & 0.993884 & 0.082513 & 0.345644 & 19.06738 \\
\hline $\mathbf{0 . 6 2 8}$ & $\mathbf{6}$ & $\mathbf{2}$ & $\mathbf{7}$ & $\mathbf{0 . 9 9 0 8 9 1}$ & $\mathbf{0 . 0 6 9 4}$ & $\mathbf{0 . 3 4 1 8 4 8}$ & $\mathbf{1 8 . 8 7 2 8 8}$ \\
\hline 0.628 & 6 & 3 & 6 & 0.988892 & 0.094561 & 0.352229 & 19.40374 \\
\hline 0.942 & 6 & 1 & 4 & 0.994355 & 0.096367 & 0.627973 & 32.12773 \\
\hline 0.942 & 6 & 2 & 3 & 0.990188 & 0.073342 & 0.615057 & 31.59389 \\
\hline 1.257 & 6 & 1 & 3 & 0.98956 & 0.029551 & 0.782382 & 38.039 \\
\hline 1.257 & 6 & 2 & 2 & 0.984922 & 0.04301 & 0.797415 & 38.56937 \\
\hline 1.257 & 6 & 3 & 2 & 0.976414 & 0.042427 & 0.80418 & 38.80555 \\
\hline 1.571 & 6 & 1 & 2 & 0.987411 & 0.020331 & 0.87789 & 41.27956 \\
\hline 1.571 & 6 & 2 & 1 & 0.987682 & 0.085308 & 0.94084 & 43.25406 \\
\hline 2.356 & 6 & 1 & 1 & 0.979583 & 0.007754 & 0.87796 & 41.28185 \\
\hline 3.141 & 6 & 1 & 1 & 0.94254 & 0.000104 & 0.806676 & 38.89227 \\
\hline 0.628 & 8 & 1 & 10 & 0.997999 & 0.082513 & 0.349281 & 19.25332 \\
\hline 0.628 & 8 & 2 & 7 & 0.996988 & 0.0694 & 0.344724 & 19.02029 \\
\hline 0.628 & 8 & 3 & 6 & 0.996287 & 0.094561 & 0.35461 & 19.52503 \\
\hline 0.942 & 8 & 1 & 4 & 0.998156 & 0.096367 & 0.637057 & 32.49947 \\
\hline
\end{tabular}




\begin{tabular}{|c|c|c|c|c|c|c|c|}
\hline 0.942 & 8 & 2 & 3 & 0.996745 & 0.073342 & 0.622145 & 31.88762 \\
\hline 0.942 & 8 & 3 & 3 & 0.994723 & 0.069904 & 0.621193 & 31.84825 \\
\hline 1.257 & 8 & 1 & 3 & 0.996547 & 0.029551 & 0.795917 & 38.51689 \\
\hline 1.257 & 8 & 2 & 2 & 0.994931 & 0.04301 & 0.808522 & 38.95631 \\
\hline 1.257 & 8 & 3 & 2 & 0.991854 & 0.042427 & 0.810646 & 39.02981 \\
\hline 1.571 & 8 & 1 & 2 & 0.995815 & 0.020331 & 0.899471 & 41.97047 \\
\hline 1.571 & 8 & 2 & 1 & 0.995867 & 0.085308 & 0.963606 & 43.93818 \\
\hline 2.356 & 8 & 1 & 1 & 0.993098 & 0.007754 & 0.92662 & 42.81881 \\
\hline 2.356 & 8 & 2 & 1 & 0.980968 & 0.003885 & 0.934455 & 43.05938 \\
\hline 3.141 & 8 & 1 & 1 & 0.979591 & 0.000104 & 0.875037 & 41.18714 \\
\hline 3.927 & 8 & 1 & 1 & 0.95415 & 0.000001 & 0.823638 & 39.47617 \\
\hline 0.628 & 10 & 1 & 10 & 0.999168 & 0.082513 & 0.351243 & 19.35349 \\
\hline 0.628 & 10 & 2 & 7 & 0.998741 & 0.0694 & 0.346449 & 19.10858 \\
\hline 0.628 & 10 & 3 & 6 & 0.99844 & 0.094561 & 0.356208 & 19.60629 \\
\hline 0.942 & 10 & 1 & 4 & 0.999233 & 0.096367 & 0.641763 & 32.69085 \\
\hline 0.942 & 10 & 2 & 3 & 0.998637 & 0.073342 & 0.626207 & 32.05508 \\
\hline 0.942 & 10 & 3 & 3 & 0.997769 & 0.069904 & 0.624472 & 31.98364 \\
\hline 1.257 & 10 & 1 & 3 & 0.998556 & 0.029551 & 0.803255 & 38.77335 \\
\hline 1.257 & 10 & 2 & 2 & 0.997864 & 0.04301 & 0.81516 & 39.18554 \\
\hline 1.257 & 10 & 3 & 2 & 0.996523 & 0.042427 & 0.815807 & 39.20782 \\
\hline 1.571 & 10 & 1 & 2 & 0.998246 & 0.020331 & 0.91099 & 42.3332 \\
\hline 1.571 & 10 & 2 & 1 & 0.99826 & 0.085308 & 0.975813 & 44.29865 \\
\hline 1.571 & 10 & 3 & 1 & 0.99717 & 0.020331 & 0.911993 & 42.36461 \\
\hline 2.356 & 10 & 1 & 1 & 0.997085 & 0.007754 & 0.952281 & 43.59981 \\
\hline 2.356 & 10 & 2 & 1 & 0.991717 & 0.003885 & 0.953725 & 43.64316 \\
\hline 3.141 & 10 & 1 & 1 & 0.991174 & 0.000104 & 0.914163 & 42.43241 \\
\hline 3.141 & 10 & 2 & 1 & 0.975931 & 0.000019 & 0.928394 & 42.87343 \\
\hline 3.141 & 10 & 3 & 1 & 0.963418 & 0.000019 & 0.940453 & 43.2423 \\
\hline 3.927 & 10 & 1 & 1 & 0.979577 & 0.000001 & 0.874959 & 41.1846 \\
\hline 4.712 & 10 & 1 & 2 & 0.896855 & 0.000072 & 0.893001 & 41.76487 \\
\hline 0.628 & 12 & 1 & 10 & 0.999595 & 0.082513 & 0.352389 & 19.41191 \\
\hline 0.628 & 12 & 2 & 7 & 0.999386 & 0.0694 & 0.3475 & 19.16233 \\
\hline 0.628 & 12 & 3 & 6 & 0.999237 & 0.094561 & 0.357221 & 19.65782 \\
\hline 0.942 & 12 & 1 & 4 & 0.999627 & 0.096367 & 0.644462 & 32.80026 \\
\hline 0.942 & 12 & 2 & 3 & 0.999335 & 0.073342 & 0.628641 & 32.15516 \\
\hline 0.942 & 12 & 3 & 3 & 0.998905 & 0.069904 & 0.626605 & 32.07147 \\
\hline 1.257 & 12 & 1 & 3 & 0.999296 & 0.029551 & 0.807555 & 38.92278 \\
\hline 1.257 & 12 & 2 & 2 & 0.998954 & 0.04301 & 0.819214 & 39.32481 \\
\hline 1.257 & 12 & 3 & 2 & 0.998285 & 0.042427 & 0.819287 & 39.32732 \\
\hline 1.571 & 12 & 1 & 2 & 0.999144 & 0.085308 & 0.982952 & 44.50741 \\
\hline
\end{tabular}




\begin{tabular}{|l|l|l|l|c|c|c|c|}
\hline 1.571 & 12 & 2 & 1 & 0.999148 & 0.085308 & 0.982946 & 44.50726 \\
\hline 2.356 & 12 & 1 & 1 & 0.99857 & 0.007754 & 0.967185 & 44.04432 \\
\hline 2.356 & 12 & 2 & 1 & 0.995871 & 0.003885 & 0.966044 & 44.01053 \\
\hline 3.141 & 12 & 1 & 1 & 0.995615 & 0.000104 & 0.93794 & 43.16579 \\
\hline 3.141 & 12 & 2 & 1 & 0.987697 & 0.000019 & 0.94541 & 43.39264 \\
\hline 3.927 & 12 & 1 & 1 & 0.989686 & 0.000001 & 0.907806 & 42.23335 \\
\hline 3.927 & 12 & 2 & 1 & 0.972094 & 0.000001 & 0.924234 & 42.74516 \\
\hline 4.712 & 12 & 1 & 4 & 0.848162 & 0.000067 & 1.010552 & 45.3007 \\
\hline
\end{tabular}

Table - 4: Sample size for DSP $(0,1)$ using Minimum Angle Method for specified values of k when the life time of the items follows Gamma Distribution

\begin{tabular}{|c|c|c|c|c|c|c|c|}
\hline $\mathbf{t} / \boldsymbol{\lambda}_{\mathbf{0}}$ & $\boldsymbol{\lambda} / \boldsymbol{\lambda}_{\mathbf{0}}$ & $\mathbf{k}$ & $\mathbf{n}$ & $\mathbf{L}\left(\mathbf{p}_{\mathbf{1}}\right)$ & $\mathbf{L}\left(\mathbf{p}_{\mathbf{2}}\right)$ & $\tan \boldsymbol{\theta}$ & $\boldsymbol{\Theta}$ \\
\hline 0.628 & 4 & 2 & 18 & 0.950122 & 0.092504 & 0.140036 & 7.971624 \\
\hline 0.628 & 4 & 1 & 28 & 0.958916 & 0.091065 & 0.138385 & 7.87882 \\
\hline 0.942 & 4 & 1 & 14 & 0.951792 & 0.089443 & 0.254165 & 14.2606 \\
\hline 0.628 & 6 & 1 & 28 & 0.990327 & 0.091065 & 0.14022 & 7.981991 \\
\hline $\mathbf{0 . 6 2 8}$ & $\mathbf{6}$ & $\mathbf{2}$ & $\mathbf{1 8}$ & $\mathbf{0 . 9 8 8 0 0 6}$ & $\mathbf{0 . 0 9 2 5 0 4}$ & $\mathbf{0 . 1 4 0 8 0 9}$ & $\mathbf{8 . 0 1 5 0 6 4}$ \\
\hline 0.628 & 6 & 3 & 17 & 0.982806 & 0.092828 & 0.141683 & 8.064164 \\
\hline 0.942 & 6 & 1 & 14 & 0.988232 & 0.089443 & 0.257913 & 14.46213 \\
\hline 0.942 & 6 & 2 & 9 & 0.985213 & 0.092765 & 0.259745 & 14.56054 \\
\hline 0.942 & 6 & 3 & 9 & 0.976669 & 0.089443 & 0.261274 & 14.64258 \\
\hline 1.257 & 6 & 2 & 6 & 0.979907 & 0.07688 & 0.37514 & 20.56306 \\
\hline 1.257 & 6 & 1 & 9 & 0.985163 & 0.078344 & 0.373571 & 20.48425 \\
\hline 1.257 & 6 & 3 & 6 & 0.968636 & 0.070409 & 0.377144 & 20.66367 \\
\hline 1.571 & 6 & 1 & 6 & 0.984138 & 0.088316 & 0.487605 & 25.99408 \\
\hline 1.571 & 6 & 2 & 4 & 0.978179 & 0.088142 & 0.490774 & 26.14061 \\
\hline 1.571 & 6 & 3 & 4 & 0.96611 & 0.081815 & 0.493961 & 26.28757 \\
\hline 2.356 & 6 & 1 & 3 & 0.980345 & 0.098071 & 0.70529 & 35.19494 \\
\hline 3.141 & 6 & 1 & 2 & 0.973381 & 0.084701 & 0.814165 & 39.15128 \\
\hline 0.628 & 8 & 1 & 28 & 0.996705 & 0.091065 & 0.141646 & 8.062078 \\
\hline 0.628 & 8 & 2 & 18 & 0.995882 & 0.092504 & 0.142 & 8.081996 \\
\hline 0.628 & 8 & 3 & 17 & 0.993998 & 0.092828 & 0.142349 & 8.10154 \\
\hline 0.942 & 8 & 1 & 14 & 0.995917 & 0.092765 & 0.261866 & 14.67433 \\
\hline 0.942 & 8 & 2 & 9 & 0.99482 & 0.092765 & 0.262185 & 14.6914 \\
\hline 0.942 & 8 & 3 & 9 & 0.991638 & 0.082397 & 0.260112 & 14.58024 \\
\hline 1.257 & 8 & 1 & 9 & 0.994752 & 0.078344 & 0.378374 & 20.72535 \\
\hline 1.257 & 8 & 2 & 6 & 0.992795 & 0.07688 & 0.378578 & 20.73555 \\
\hline 1.257 & 8 & 3 & 6 & 0.988449 & 0.070409 & 0.377702 & 20.69163 \\
\hline 1.571 & 8 & 1 & 6 & 0.99431 & 0.081815 & 0.491747 & 26.18553 \\
\hline 1.571 & 8 & 2 & 4 & 0.99205 & 0.088316 & 0.496514 & 26.40503 \\
\hline & & & & & & & \\
\hline & & & & & & & \\
\hline
\end{tabular}




\begin{tabular}{|c|c|c|c|c|c|c|c|}
\hline 1.571 & 8 & 3 & 4 & 0.987296 & 0.081815 & 0.495556 & 26.361 \\
\hline 2.356 & 8 & 1 & 3 & 0.992703 & 0.098071 & 0.722234 & 35.83809 \\
\hline 2.356 & 8 & 2 & 3 & 0.979692 & 0.032876 & 0.682428 & 34.31072 \\
\hline 3.141 & 8 & 1 & 2 & 0.989772 & 0.032363 & 0.795229 & 38.49275 \\
\hline 3.141 & 8 & 2 & 2 & 0.972033 & 0.032363 & 0.810242 & 39.01583 \\
\hline 3.927 & 8 & 1 & 2 & 0.978395 & 0.026441 & 0.856633 & 40.58445 \\
\hline 4.712 & 8 & 1 & 2 & 0.961331 & 0.007635 & 0.870691 & 41.04579 \\
\hline 0.628 & 10 & 1 & 28 & 0.998596 & 0.091065 & 0.142489 & 8.109444 \\
\hline 0.628 & 10 & 2 & 18 & 0.998239 & 0.092504 & 0.142772 & 8.125309 \\
\hline 0.628 & 10 & 3 & 17 & 0.997413 & 0.092828 & 0.142953 & 8.135509 \\
\hline 0.942 & 10 & 1 & 14 & 0.998241 & 0.089443 & 0.262708 & 14.71947 \\
\hline 0.942 & 10 & 2 & 9 & 0.997758 & 0.092765 & 0.263813 & 14.77865 \\
\hline 0.942 & 10 & 3 & 9 & 0.99634 & 0.082397 & 0.261229 & 14.64016 \\
\hline 1.257 & 10 & 1 & 9 & 0.997713 & 0.078344 & 0.38135 & 20.87433 \\
\hline 1.257 & 10 & 2 & 6 & 0.996839 & 0.07688 & 0.381105 & 20.86209 \\
\hline 1.257 & 10 & 3 & 6 & 0.994864 & 0.070409 & 0.379251 & 20.76929 \\
\hline 1.571 & 10 & 1 & 6 & 0.997498 & 0.088316 & 0.499933 & 26.56197 \\
\hline 1.571 & 10 & 2 & 4 & 0.996477 & 0.088142 & 0.500399 & 26.58332 \\
\hline 1.571 & 10 & 3 & 4 & 0.99429 & 0.088316 & 0.501703 & 26.64305 \\
\hline 2.356 & 10 & 1 & 3 & 0.99672 & 0.098071 & 0.73232 & 36.21608 \\
\hline 2.356 & 10 & 2 & 3 & 0.99064 & 0.032876 & 0.68712 & 34.49375 \\
\hline 3.141 & 10 & 1 & 2 & 0.9953 & 0.084701 & 0.857484 & 40.61255 \\
\hline 3.141 & 10 & 2 & 2 & 0.986754 & 0.032363 & 0.818139 & 39.28793 \\
\hline 3.141 & 10 & 3 & 2 & 0.979195 & 0.032062 & 0.824408 & 39.50245 \\
\hline 3.927 & 10 & 1 & 2 & 0.989765 & 0.026441 & 0.875427 & 41.19977 \\
\hline 3.927 & 10 & 2 & 2 & 0.972015 & 0.009439 & 0.876107 & 41.22183 \\
\hline 4.712 & 10 & 1 & 2 & 0.981111 & 0.007635 & 0.890692 & 41.6912 \\
\hline 4.712 & 10 & 2 & 2 & 0.950089 & 0.002636 & 0.915156 & 42.46339 \\
\hline 0.628 & 12 & 1 & 28 & 0.999307 & 0.091065 & 0.143004 & 8.138349 \\
\hline 0.628 & 12 & 2 & 18 & 0.999128 & 0.092504 & 0.143259 & 8.152669 \\
\hline 0.628 & 12 & 3 & 17 & 0.998713 & 0.092828 & 0.143376 & 8.159233 \\
\hline 0.942 & 12 & 1 & 14 & 0.999124 & 0.089443 & 0.26382 & 14.77903 \\
\hline 0.942 & 12 & 2 & 9 & 0.998881 & 0.092765 & 0.264858 & 14.83462 \\
\hline 0.942 & 12 & 3 & 9 & 0.998163 & 0.082397 & 0.262067 & 14.68509 \\
\hline 1.257 & 12 & 1 & 9 & 0.998853 & 0.078344 & 0.383214 & 20.96752 \\
\hline 1.257 & 12 & 2 & 6 & 0.998409 & 0.07688 & 0.382789 & 20.94632 \\
\hline 1.257 & 12 & 3 & 6 & 0.997395 & 0.070409 & 0.380536 & 20.83361 \\
\hline 1.571 & 12 & 1 & 6 & 0.998737 & 0.088142 & 0.502741 & 26.69055 \\
\hline 1.571 & 12 & 2 & 4 & 0.998215 & 0.088142 & 0.50303 & 26.70375 \\
\hline 2.356 & 12 & 1 & 3 & 0.998319 & 0.098071 & 0.738606 & 36.44979 \\
\hline
\end{tabular}




\begin{tabular}{|c|c|c|c|c|c|c|c|}
\hline 2.356 & 12 & 2 & 3 & 0.995135 & 0.032876 & 0.691008 & 34.64479 \\
\hline 3.141 & 12 & 1 & 2 & 0.997555 & 0.084701 & 0.867738 & 40.94942 \\
\hline 3.141 & 12 & 2 & 2 & 0.992987 & 0.032062 & 0.82433 & 39.49977 \\
\hline 3.141 & 12 & 3 & 2 & 0.988802 & 0.032062 & 0.827935 & 39.62255 \\
\hline 3.927 & 12 & 1 & 2 & 0.994567 & 0.026441 & 0.88805 & 41.60667 \\
\hline 3.927 & 12 & 2 & 2 & 0.984761 & 0.009439 & 0.881498 & 41.3961 \\
\hline 3.927 & 12 & 2 & 2 & 0.984761 & 0.009439 & 0.881498 & 41.3961 \\
\hline 4.712 & 12 & 1 & 2 & 0.989768 & 0.007635 & 0.905244 & 42.15279 \\
\hline 4.712 & 12 & 2 & 2 & 0.972023 & 0.002636 & 0.917147 & 42.52541 \\
\hline
\end{tabular}

Table - 5: comparison of results for $\operatorname{DSP}(0,1)$ for different lifetime distributions

\begin{tabular}{|c|l|c|c|c|c|c|}
\hline S.No & \multicolumn{1}{|c|}{ Distribution } & $\begin{array}{c}\text { Producer's } \\
\text { risk }\end{array}$ & Consumer's risk & $\mathbf{k}$ & $\mathbf{n}_{\mathbf{1}}$ & $\mathbf{n}_{\mathbf{2}}$ \\
\hline 1 & Rayleigh & 0.0097 & 0.057 & 2 & 15 & 30 \\
\hline 2 & Generalized Exponential & 0.0225 & 0.086 & 3 & 10 & 30 \\
\hline 3 & Weibull & 0.009 & 0.069 & 2 & 7 & 14 \\
\hline 4 & Gamma & 0.0119 & 0.092 & 2 & 18 & 36 \\
\hline
\end{tabular}

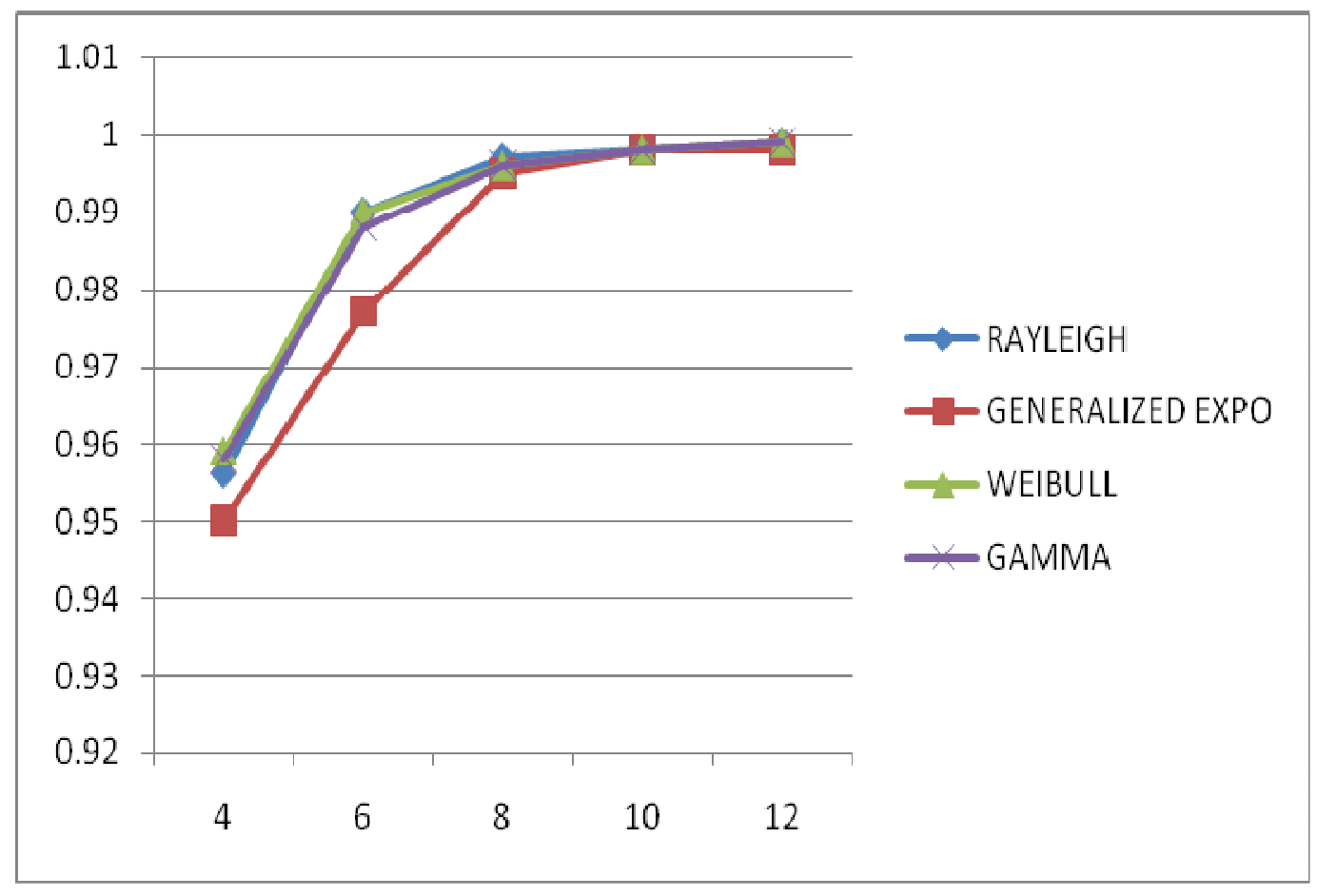

Fig - 1: OC curve for DSP(0,1) various lifetime distributions 


\section{CONCLUSIONS}

In this paper designing of DSP $(0,1)$ sampling plan for truncated life tests by using minimum angle method is presented. The minimum sample sizes for various values of $\lambda / \lambda_{0}$ and different experiment times are calculated assuming that the lifetime of the test items follows different lifetime distributions. When the table values (Table 1 to Table 4) are compared one can say that weibull distribution is comparatively better than other distributions. It can be seen that by applying minimum angle method minimizes simultaneously the consumer's and producer's risk. This minimum angle method plan provides better discrimination of accepting good lots

\section{REFERENCES}

[1]. Goode, H.P., \& Kao, J.H.K (1961). Sampling plans based on the distribution. In Proceeding of the Seventh National Symposium on Reliability and Quality Control (pp. 24-40), Philadelphia.

[2]. Balizi, A (2003). Acceptance sampling based on truncated life tests in the Pareto distribution of the second kind. Advances and Applications in Statistics, 3 (1), 33-48.

[3]. Balizi \& EI Masri (2004), Acceptance sampling based on truncated life tests in the Birnbaum Saunders model. Risk Analysis, 24(6), 1453.

[4]. Rosaiah, K., Kantam, R. R. L. (2005). Acceptance sampling based on inverse Rayleigh distribution. Econo. Qual. Control 20:277-286.

[5]. Tsai, T.R., \& Shou (2006). Acceptance sampling based on truncated life for generalized Rayleigh Distribution. Journal of applied statistics, 33 (6), $595-600$

[6]. Muhammad Aslam, (2007). Double Acceptance Sampling Based on Truncated Life tests in Rayleigh Distribution. European Journal of Scientific Research, Vol.17 pp.605-610.

[7]. Bush N. Leonard E.J., and Merchant M.Q.M.Jr., (1953) A Method od Single and Double Sampling OC curves Utilizing the Tangent of the Point of the Inflexion, (ENASR), No, PR-7, $1-77$.

[8]. Srinivasa Rao (2009) "Reliability test plans for Marshall Olkin extended exponential distribution" Applied mathematical sciences, Vol. 3 (2009), Number 55, 2745-2755 